\title{
JWeb: an HDM Environment for Fast Development of Web Applications
}

\author{
Mario Bochicchio ${ }^{1}$, Roberto Paiano ${ }^{1,}$ Paolo Paolini ${ }^{2,1}$ \\ ${ }^{1}$ Telemedia Lab, University of Lecce, Italy, \{bomal, rpaiano\}@ingle01.unile.it \\ ${ }^{2}$ Hypermedia Open Center, Politecnico di Milano, Italy, paolini@elet.polimi.it
}

\begin{abstract}
JWeb is a development environment for complex $W W W$ applications, based upon the HDM model. JWeb (entirely written in Java) consists of two different parts: the development environment and the execution environment. The development environment allows the definition of the conceptual structure of the application, the creation of a supporting DataBase and the DataBase population. The execution environment allows the running of the application, possibly after some customization operations. We envision three main ways of using JWeb:

- as a training tool, for students and practitioners;

- as a fast prototyping tool, to test different design hypothesis, and to come up with a final design;

- as a tool for fast development and deployment of complex, high quality, Web applications.
\end{abstract}

\section{Introduction and background}

Since a number of years the model and the methodology HDM [1-4], developed at the Hypermedia Open Center of the Politecnico di Milano, have been used to develop complex high quality Hypermedia applications.

The methodology envisions that a conceptual model of the application, a schema, is developed first, directly from the requirement analysis. The schema of an application consists of two parts: hyperbase schema and access schema. The hyperbase schema describes the basic navigational capabilities, using as ingredients Entities (organized into Entity types), and Links (organized into Link Types). The access schema, instead, describes the organizations of the access structures (collections in the HDM terminology), leading to the definition of indexes (possibly hierarchically organized), guided tours, etc.

Both schemas have a part in-the-large, which describes the overall aspects of the application, and a part in-thesmall, which provides the needed details up to the field level.

The basic development steps of an application can be summarized as it follows ${ }^{1}$ :

- $\quad$ production of an HDM schema;

\footnotetext{
${ }^{1}$ What is presented here is a very crude simplification, with respect to a complex development strategy involving more than 12 steps.
}

- development of a supporting Data Base (the logical structure of which is derived from the structure of the schema);

- insertion of the instances, i.e. the population of the Data Base with actual data;

- development of the runtime SW to actually execute the application.

A development environment supporting these steps is desirable for different purposes:

$\Rightarrow$ to provide trainees with a fast feedback for their design practice. With a supporting environment, a trainee may develop different design alternatives, and immediately visualize the implications of the different choices.

$\Rightarrow$ To allow a fast prototyping of the application.

If an HDM schema, in fact, is perfectly readable to anyone well acquainted with the model itself, it may be not fully understood by "non expert readers". Fast prototyping allows everybody to fully understand what the current design defines, allowing also discussions, changes and improvements of the design itself. Fast prototyping, in the end, improves the overall quality of the design, and therefore of the resulting application.

$\Rightarrow$ To allow, in some cases, a kind of (semi) automatic development of the final application, through a generalized HDM interpreter [5].

Automatic generation is very appealing, since it may reduce costs and time needed for developing complex applications. Only recently the standardization of the runtime environments for the Web, has made this objective seriously attainable. But there are still problems, when specific architectural requirements must be taken into account, or when high level, professional interfaces, must be considered. For such a reasons we do believe that the first two uses of JWeb (training and fast prototyping) are more important, for the time being, than automatic development.

JWeb, as a SW environment, is an offspring of the Autoweb [6,7] effort. The main differences, with respect 
to Autoweb, are a consequence of the different objectives. Autoweb pursues the goal of providing an automatic development environment; therefore it becomes more and more complex, in terms of system options or interface building. JWeb, instead, tries to remain simple and easy to use, aiming at being mainly a training and a prototyping tool.

Both Autoweb and JWeb are based upon the HDM model [1, 3, 8, 9], developed at the Politecnico di Milano, used by several organizations around the world. HDM was the first model proposed for a systematic top-down development of Hypermedia (and therefore WWW) applications. Other models have been proposed later, such as OOHDM [10] and RMM [11], all of them derived from HDM.

The structure of this paper is the following: in section 2 we describe the overall structure of the JWeb environment, and the role of each component; a few implementation details are also provided. In section 3 we describe an example of application (on the borderline between fast prototyping and final application). In section 4 we draw the conclusions and sketch the future directions for work. Section 5 is for bibliography and references.

\section{The JWeb Environment}

The overall picture of a JWeb environment is shown in fig. 1. There are five major components: the Schema Editor, the Instance Editor, the Configurator, the Generator and the Interpreter.

Let us briefly describe the nature and the role of each one of the tools:
- Schema Editor

It allows to designer to define the conceptual schema of the application. Using the conceptual primitives offered by HDM, he/she can define the Hyperbase (i.e. the core of the application) and the Access Structures (i.e. the way to initiate the navigation within the application).

- Instance Editor

It allows two different functions: the creation of a supporting relational database (the "content" database) and the population of it with actual data. The logical structure of the database is derived from the conceptual structure defined with the schema editor.

- Configurator

It allows the "customization" of the application. Customization means to select part of the schema (and therefore, part of the content database) and also the possible addition of new access structures. The output produced by the Configurator is a new schema.

- Generator

It takes as input the schema produced by the Configurator and the content database. Its output is the database actually used by the application.

- HDM-interpreter.

It is the tool that actually executes the application.

Let us provide more details about the different parts of the environment. The Schema Editor provides the capability to design the "Hyperbase in the large" [3], the "Hyperbase in the small"[3] and the "access structures"[8], according to the HDM model. The output of the tool is a complete

Development

Run-Time

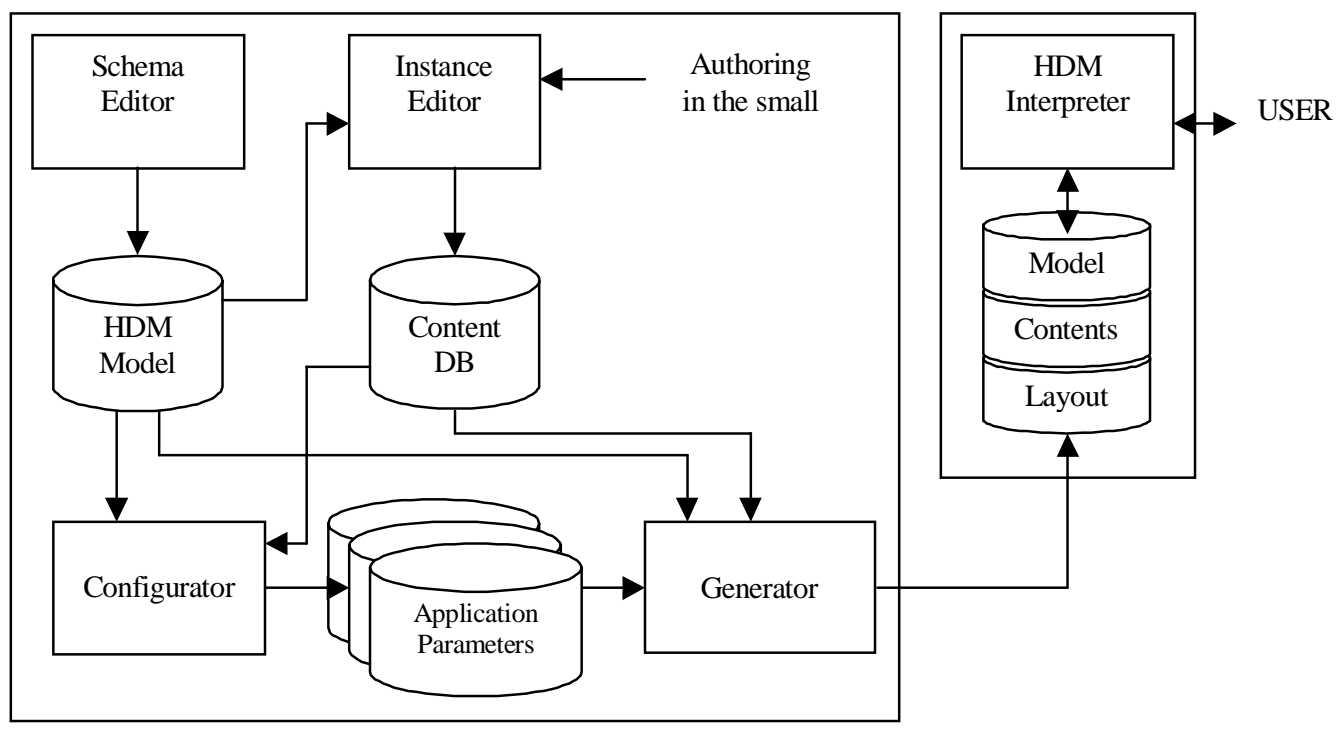

Figure 1. The JWeb environment 
definition of the application, the so-called schema. The schema is described in XML using a DTD specifically created for HDM. The Schema Editor contains an XML parser that validates the model and read/modify/save it. This solution allows to import HDM models, written using the same DTD, generated by other possible sources. The XML parser is based on a specific Stream Tokenizer initialized to recognize the XML characters. An example of this DTD, related to Application Links definition, is shown here:

\section{ACCESSTYPE2 CDATA \#REQUIRED \\ MINCARD CDATA \#REQUIRED \\ MAXCARD CDATA \#REQUIRED>}

The Instance Editor allows defining the logical schema and the physical structure of a relational Data Base, supporting the wished HDM schema. The main input for the Instance Editor is represented by the XML description of the application, generated by the Schema Editor. Additional information is provided by details about the single items of information to be handled (authoring in the small, in the HDM terminology).

The Configurator allows to define the complete schema of a specific application, that is, in general, a subset (hyperview in the HDM terminology) of the application defined with the Schema Editor. Specific types of objects (entity types, in HDM terminology), or specific slices of data (e.g. only the objects, within a given types, satisfying some specified conditions) can be selected. In addition, in order to make it possible to customize the application for specific needs, ad-hoc access structures (collections [8], in HDM terminology) can be defined. The output of the Configurator is, again, an XML description.

The Generator takes as input the XML descriptions coming from the Schema Editor and the Configurator, respectively. Its job is to produce a Data Base, subset of the one generated by the Instance Editor, and also to generate all the needed descriptions (again in XML).

The most important tool is obviously the HDM Interpreter (HDMI in the following), that allows the actual execution of an application, depicted in Fig. 2. HDMI is a Java application communicating with the Web Server (via CGI) and with the DBMS (via JDBC [10]); the job performed by the interpreter is the on-the-fly creation of the application pages when requested. The user interface of the application is rendered, on the clients, by a standard Web Browser. A number of Java applets running on the Java Virtual Machine of the browser complete the HDMI on the client side.

The most important component of HDMI is the Navigation Engine (NE), which actually implements the navigation in-the-large, i.e. the navigation from one node of the application to another node. The NE compute the actual meaning of a user navigation request, on the basis of the current situation of navigation, and by applying the HDM navigation rules over the schema of the application.

The current version of JWeb has been implemented and tested on a pool of networked Win95 workstations, using JDK 1.1.4. Moreover, we verified the compatibility with various DBMSs (MS Access, IBM DB2 and Oracle running on various Operating Systems, via JDBC/ODBC) and various Web Servers (Apache and MS Personal Web Server).

\section{An Example}

An earlier version of JWeb has been used to implement a training application (concerning advanced SW development systems), for a large industrial consortium, and a multimedia CD-ROM concerning the history of Computer Science in Italy, for the Italian National Agency of Research (CNR).

Two major applications are currently being developed,

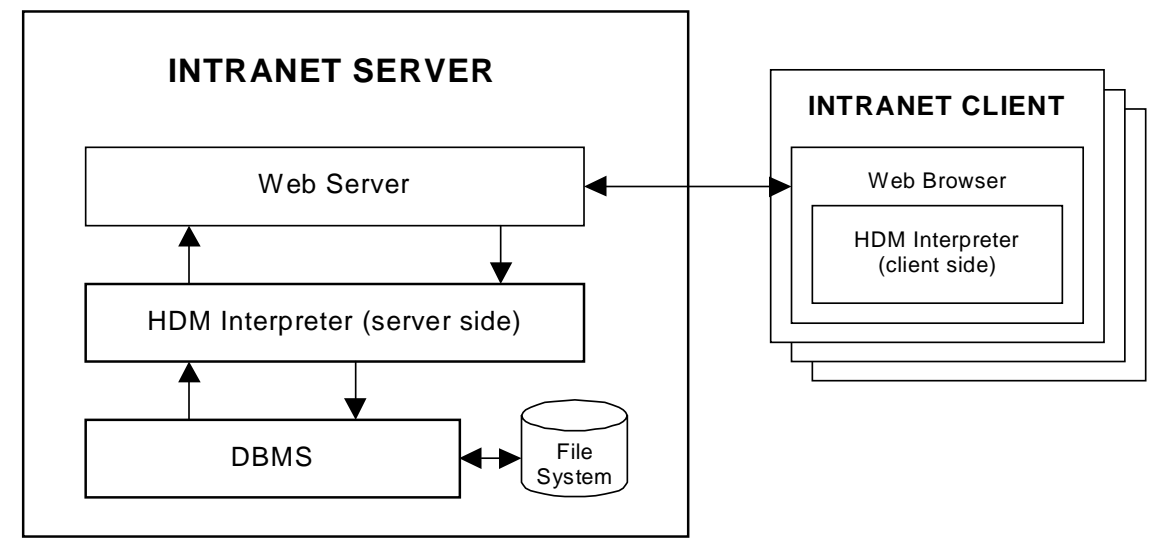

Figure 2. System level description of an on-line application based on JWeb 
both concerning multimedia catalogues for the fashion industry. One of the applications is for a small manufacturing group, active in the garment industry for youngsters. The other application is for a pool of high level pret-a-porter for men and women.

We describe, here, as an example, the first of the two applications. The goal is to develop a multimedia catalogue for supporting the sales of jeans, street ware, casual ware, etc. and related items. The application is envisioned as being delivered both online, and offline (as a CD-ROM). In both cases the application must be based upon Internet technology, i.e. it must be possible to execute it by using a standard browser equipped with standard plug-ins (for the multimedia part).

Since different uses are envisioned (e.g. accessible from home, accessible from retailers, accessible at fairs and special situations, etc.) it is very important that the same content can be organized in different ways, specially tuned for the different purposes.

The HDM schema for the application consists of eight Entity types:

- Model: represent a specific model of casual clothing. It is strictly related to fit. Several "models" may have the same fit.

- Style: represents a collection of models related to the same "world". A reference world (music, images, etc.) is also provided for each style. Young men or women should identify themselves in a scenario (pictures from a given life-style, music, ...) to choose the specific model of clothing.

- Fabric: the material of which a number of products are made. Text and images are used to illustrate it. Each model can be made in several fabrics and with several finishes.

- Article: represents a model of clothing in a specific fabric and color. It is a real piece that can be bought. It carries, among with pictures and text, information about its price, availability or delivery terms.

- $\quad$ Fit: each model has a specific fit (like slim, baggy, normal and so on).

- Accessory: it represents the details of a garment, such as like zip or button fly, front slant pockets, back square pockets, coin pockets, hammer loop, key loop and so on.

- Size Table: represent the different sizes available for the specific article. It also has a design that explain the specific size implementation (measures).

- Other: it contains additional multimedia material, not strictly related to the business, but important in the overall marketing approach of the application.

The access structures, provided by HDM, are either intensional or extensional. The intensional structures can be automatically created by using the attributes stored into the database. The collection "all the shirts ordered by fabric" is an example of intensional access structure.
The extensional access structures are created, instead, to take into account more subjective points of view. For example, "clothing for free time" is an extensional collection of specific models, defined for a specific marketing purpose. Extensional access structures are defined during the configuration phase.

The visual layout of the user interface, for this application, has been implemented by using Director and Shockwave. In the initial phase of prototyping, a very crude layout was plugged in, just to check the overall working of the application.

After a while a professional layout was developed by professionals, and in a few days it was plugged in, without having to revise the Software.

An initial version of the application will be delivered to a selected number of points of sale, by the end of March 99. At the same time a different configuration of the application (obtained with the configurator) is being tested at fairs and commercial exhibitions.

\section{Conclusions and future work}

It is an obvious observation the fact that we need to improve the quality of hypermedia applications (both offline and online), at the same time reducing the time and the costs required for their development. Since a number of years we have acknowledged the fact that having a structured approach to design, using a model, such as HDM [1-4], is an important step in this direction.

Our design experience (over a number of years) has also shown that some type of support to design and implementation is heavily needed. The ancestor of a support system was the environment developed by the HYTEA project, and then the tools provided by the MINERS [12] and HIFI [13] projects, all of them partially funded by the European Commission. All these above environments were targeted toward offline applications. Recently, however, the interest for the development of WWW applications has become predominant. Systems such as Autoweb [6,7] based on HDM-lite, and RMM based upon a derivative of HDM, aim at improving the productivity of WWW application development.

JWeb is an important step for a number of reasons:

- It makes use of a model, extended HDM, much more powerful than the models used by other systems.

- It consists of a set of independently usable modules.

- All the internal descriptions of structures and data are based upon XML; all the SW is written in Java; therefore the whole system is very portable.

- The notion of hyperview is fully exploited, in order to maximize the reuse of the multimedia content, for different purposes and for different situations.

- Through the use of Shockwave for the layout, it allows the use of very high quality visual communications, suitable also in specific sales and marketing situation. 
Our future work mainly consists in completing the development of the whole environment and testing the environment itself. The initial testing for fast prototyping consists in completing the two applications above described. For both of them, some kind of final delivery is also considered.

As far as training is concerned, 200 students, of graduate classes in the University of Lecce, Politecnico of Milano and University of the Italian Switzerland in Lugano, will use it.

A special version of the JWeb environment will be also included in the SITMOON environment, supporting the work of publishing companies, developed within a project funded by the European Commission.

In more strategic sense, a full revision of the overall JWeb environment is planned and is supposed to be completed by the fall of '99. The revision will include an improved version of each tool and a more flexible delivery environment, moreover the role of hyperviews will be greatly enhanced. The idea is that, in the end, complex application environments will be developed, with specific applications (either on-line or off-line) derived from it.

In order to support this more ambitious point of view, also enhancements and extension to the model are needed. For such a reason a new version of HDM, labeled HDM2000, is envisioned by the end of '99. The revision of the model and of the JWeb environment is a joint effort of Politecnico di Milano (at the campuses of Como, Bovisa and Milano), University of Lecce (engineering school), University of Lugano (communication School) and MIP (an industrial consortium, specialized in technology transfer to small medium companies).

Acknowledgment: the authors wish to thank $P$. Fraternali, the main driving force behind Autoweb, from which JWeb was initially derived, and the JWeb development team.

\section{Bibliography}

[1] F. Garzotto, P. Paolini, and D. Schwabe, "HDM - A Model Based Approach to Hypermedia Application Design", ACM Transactions on Information Systems, Vol. 11, N. 1, pp. 1-26, Jan. 1993.

[2] F. Garzotto, L. Mainetti, and P. Paolini, "Information Reuse in Hypermedia Applications", Hypertext 1996, Washington DC, USA, pp. 93-104

[3] F. Garzotto, L. Mainetti, and P. Paolini, "Hypermedia Application Design: a Structured Approach", in J.W. Schuler, N. Hannemann, and N. Streitz Eds., "Designing User Interfaces for Hypermedia", Springer Verlag, 1995.

[4] D. Schwabe, and G. Rossi, "The Object-Oriented Hypermedia Design Model", Communications of the ACM, Vol. 38, N. 8, pp.45-46, Aug. 1995.

[5] M.A. Bochicchio, and P. Paolini, "An HDM Interpreter for On-Line Tutorials", MultiMedia Modeling '98 Lausanne, Switzerland, Oct. 1998, pp.184-190.

[6] P. Fraternali, and P. Paolini "A Conceptual Model and a Tool Environment for Developing More Scalable, Dynamic, and Customizable Web Applications". EDBT 1998, pp. 421-435.

[7] P. Fraternali, and P. Paolini, "The Autoweb System"; submitted for publication

[8] F. Garzotto, L. Mainetti, and P. Paolini, "Adding Multimedia Collections to the Dexter Model". ECHT 1994, pp. 70-80

[9] F. Garzotto, L. Mainetti, and P. Paolini "Hypermedia Design, Analysis, and Evaluation Issues", Communications of the ACM Vol.38, No.8, pp.74-86, Aug. 1995.

[10] D. Schwabe, and G. Rossi, "The Object-Oriented Hypermedia Design Model", Communications of the ACM, Vol. 38, N. 8, pp.45-46, Aug. 1995.

[11]T. Isakowitz, E.A. Stohr, and P. Balasubramanian, "RMM: a Methodology for Structured Hypermedia Design", Communications of the ACM Vol.38, No.8, Aug. 1995, pp. 3344.

[12] MINERS Projects reports, available from Politecnico di Milano and from EDS-Italy corporate research center in Roma, Italy.

[13] U. Cavallaro, F. Garzotto, P. Paolini, and D. Totaro, "HIFI: Hypertext Interface for Information Systems", IEEE Software Vol. 10, N. 6,pp. 48-51, Nov. 1993. 